\title{
Desarrollo de un modelo de gestión de la inteligencia organizacional para la compañía Gráficas Modernas S.A.*
}

Development of an Organizational Intelligence Management Model for Gráficas Modernas Inc. Desenvolvimento de um modelo de gestão da inteligência organizacional para a companhia Gráficas Modernas S.A.

\author{
Jonathan Lozano Oviedo ** \\ Carlos Hernán González Campo ${ }^{\star \star *}$
}

Fecha de recibido: 23 de agosto de 2014

Fecha de aprobado: 20 de enero de 2015

Doi: dx.doi.org/10.12804/rev.univ.empresa.29.2015.03

Para citar este artículo: Lozano Oviedo, J., \& González Campo, C. H. (2015). Desarrollo de un modelo de gestión de la inteligencia organizacional para la compañía gráficas modernas S.A. Universidad \& Empresa, 17(29), 63-91. Doi: dx.doi.org/10.12804/rev.univ.empresa.29.2015.03

\footnotetext{
Este artículo es producto del proyecto de investigación "La Inteligencia Organizacional (IO) en la compañía Gráficas Modernas S.A.”. Fue realizado como requisito para optar al título de magíster en Administración de la Universidad del Valle (Colombia).

** Magíster en Administración de Empresas (mba) e Ingeniero Industrial de la Universidad del Valle (Cali, Colombia). Analista técnico del Departamento de Actuaría de Coomeva Corredores de Seguros (Cali, Colombia). Correo electrónico: jonathanlozanoviedo@gmail.com

*** Doctor en Administración de la Universidad EAFIT (Medellín, Colombia), Magíster en Ciencias de la Organización de la Universidad del Valle (Cali, Colombia). Especialista en Talento Humano y en Mercadeo de la Universidad Libre de Colombia (Cali), Economista de la Universidad del Valle (Cali, Colombia). Profesor Titular del Departamento de Administración y Organizaciones de la Facultad de Ciencias de la Administración de la Universidad del Valle (Cali, Colombia). Correo electrónico: carlosh.gonzalez@correounivalle.edu.co
} 


\section{RESUMEN}

La inteligencia organizacional (IO) es un tema que ha sido investigado desde la literatura administrativa, lo que es interesante para el contexto organizacional, dadas las implicaciones que una mejora de la Io puede tener en el éxito, la conservación en el tiempo y el desarrollo de una compañía. Este artículo incorpora los resultados de una investigación sobre la Io realizada en el contexto de la compañía Gráficas Modernas S.A. El presente trabajo se propuso desarrollar un modelo de gestión de la Io para esta empresa.

Palabras clave: gestión del conocimiento, inteligencia organizacional, modelos de gestión, organizaciones que aprenden.

\section{ABSTRACT}

Organizational Intelligence (OI) is a subject that has been researched by the management literature. This is relevant for the organizational context, given the implications that an improvement in ol can mean for the success, the conservation in time, and the development of a company. This article includes the results of an or research carried out within the context of the company Gráficas Modernas Inc. The intention of this paper is to develop an oI management model for this enterprise.

Keywords: Knowledge management, learning organizations, management models, organizational intelligence.

\section{RESUMO}

A inteligência organizacional (IO) é um tema que tem sido pesquisado desde a literatura administrativa. Isto é interessante para o contexto organizacional, dadas as implicações que uma melhora da Io pode ter no sucesso, a conservação no tempo e o desenvolvimento de uma companhia. Este artigo incorpora os resultados de uma pesquisa sobre a Io realizada no contexto da companhia Gráficas Modernas S.A. Este trabalho se propôs desenvolver um modelo de gestão da Io para esta empresa.

Palavras-chave: gestão do conhecimento, inteligência organizacional, modelos de gestão, organizações que aprendem. 


\section{INTRODUCCIÓN}

La inteligencia organizacional (IO) es un tema de investigación bastante amplio y dinámico. Considera una gran cantidad de elementos organizacionales, tangibles e intangibles e interrelacionados sistémicamente, que buscan garantizar el logro de un propósito o una misión determinada. En la Io se resalta la importancia de la gestión permanente en temas clave como la percepción del entorno interno y externo de la organización, las capacidades individuales, grupales y colectivas para aprender y crear e innovar, la creación y gestión del conocimiento organizacional, la toma de decisiones acertadas, las capacidades de respuesta y resolución de problemas, la innovación y el desempeño organizacional (en sus perspectivas financiera, comercial, de servicio, de procesos internos y del aprendizaje organizacional) y la inteligencia emocional, entre otros.

El objetivo general de esta investigación es desarrollar un modelo de gestión de la Io para la organización Gráficas Modernas S.A. La metodología empleada se desplegó en cuatro grandes fases: 1) análisis y revisión de las teorías y modelos existentes acerca de la IO, 2) diagnóstico de la IO en la empresa Gráficas Modernas S.A., el cual fue realizado mediante dos técnicas de investigación, en particular, la encuesta y la entrevista, 3) desarrollo empírico o, en otras palabras, identificación del componente empírico emergente de la IO; esto según el contexto empresarial y las particularidades empresariales, y 4) elaboración del modelo de gestión de la Io para la organización.

Esta investigación pretende aportar al avance de la IO, en particular, a través de la elaboración de un modelo de gestión de la io para una empresa del sector de artes gráficas. Esto fundamentándose en una propuesta de definición de la Io. Se considera que este es un aporte visible para la academia y el mundo empresarial, dados 1) la constitución de una definición propia de la IO y 2) el hecho de que, en la revisión de literatura realizada, no se encontraron publicaciones relativas a desarrollos de modelos de gestión de la Io para una empresa particular en este mismo sector y localidad.

\section{METODOLOGÍA}

Se realizó inicialmente una revisión de la literatura administrativa relacionada con la Io. Gracias a esta fue posible identificar una serie de categorías de análisis o dimensiones específicas vinculadas a desarrollos previos en esta materia. Estas se presentan a continuación: 
- Dominio personal, modelos mentales, visión compartida, aprendizaje en equipo y pensamiento sistémico, como disciplinas de la organización con IO (Senge, 1990).

- Visión estratégica, apetito por el cambio, corazón, alineación, congruencia y presión por el desempeño, como dimensiones de la IO (Albrecht, 2002, 2003).

- Inteligencia emocional (Halal, 1997; Kalkan, 2005; North \& Rivas, 2008).

- Creación y gestión del conocimiento organizacional (Arévalo, 2008; Choo, 1999; Deagostini \& Comenzana, 2005; Nonaka \& Takeuchi, 2000; Pinheiro, Hernández, \& Raposo, 2007).

Fue posible también encontrar una serie de definiciones, acciones y otros aspectos relevantes vinculados directamente con la IO. Entre estos se destacan los siguientes:

- La acción de la organización evidenciada en la capacidad de responder y resolver problemas (North \& Rivas, 2008)

- La percepción del entorno interno desde la capacidad de vigilancia e interpretación del entorno interno (Choo, 1999; North \& Rivas, 2008)

- La percepción del entorno externo desde la capacidad de vigilancia e interpretación del entorno externo (Choo, 1999; Mendelson \& Ziegler, 2004; North \& Rivas, 2008)

- La capacidad de aprender y crear o innovar (North \& Rivas, 2008)

- La toma de decisiones adaptativas y generativas (Choo, 1999; Senge, 1990)

- El desempeño organizacional y los resultados innovadores (Kalkan, 2005)

En particular las categorías de análisis identificadas fueron contempladas en la metodología del proyecto de investigación. Esta estuvo soportada por una combinación de métodos y técnicas referentes, tal y como se expresa a continuación:

- Está presente el método inductivo: En el presente estudio fue utilizada la encuesta. Esta técnica de investigación permite intentar formular generalizaciones a partir de casos particulares. Ello observando características determinadas sobre los colaboradores para tratar de generalizar las de toda la población objeto de estudio. Se encuestó a todos y cada uno de los colaboradores de la organización, es decir, a treinta y ocho empleados. Esto incluyendo a los líderes de la compañía.

- Está también presente el método deductivo: Fueron entrevistados todos los colaboradores 
con cargos directivos o de liderazgo. Esto incluyendo al presidente ejecutivo. Esta actividad se realizó para tener una información concreta, complementaria y precisa en las dimensiones abordadas por esta técnica. En total siete colaboradores fueron entrevistados. Ellos conforman el cuerpo directivo de la empresa Gráficas Modernas S.A.

Las cuatro fases realizadas para llevar a cabo la investigación se presentan de manera sintética en la siguiente tabla:

Tabla 1. Fases de la investigación

\begin{tabular}{|c|c|c|}
\hline Fases & $\begin{array}{c}\text { Técnica de } \\
\text { estudio }\end{array}$ & Temas de la inteligencia organizacional \\
\hline $\begin{array}{l}\text { Fase } 1 \\
\text { Teorías y modelos } \\
\text { sobre la inteligencia } \\
\text { organizacional. } \\
\text { (Elementos teóricos que } \\
\text { facilitan la medición y } \\
\text { gestión de la Io). }\end{array}$ & $\begin{array}{l}\text { Análisis } \\
\text { documental y } \\
\text { revisión de la } \\
\text { literatura. }\end{array}$ & $\begin{array}{l}\text { - En la literatura administrativa acerca de la IO: defini- } \\
\text { ciones, características, condiciones de gestión, aspectos } \\
\text { comunicacionales, principios y componentes básicos, } \\
\text { modelos teóricos y herramientas de diagnóstico. } \\
\text { - Estado del arte (inteligencia organizacional, modelos } \\
\text { de gestión de la Io y aplicaciones de herramientas de } \\
\text { diagnóstico de IO). } \\
\text { - En Gráficas Modernas S.A.: Misión, visión, historia, } \\
\text { principales productos, organigrama y plan estratégico } \\
\text { de } 2011 \text {. }\end{array}$ \\
\hline $\begin{array}{l}\text { Fase } 2 \\
\text { Diagnóstico de la IO. } \\
\text { (Trabajo de campo: } \\
\text { diagnóstico de la Io en } \\
\text { la empresa Gráficas } \\
\text { Modernas S.A.). }\end{array}$ & $\begin{array}{c}\text { Análisis } \\
\text { documental, } \\
\text { revisión de } \\
\text { la literatura, } \\
\text { encuestas a los } \\
\text { colaboradores } \\
\text { (censo), } \\
\text { entrevistas } \\
\text { a todos los } \\
\text { líderes de la } \\
\text { organización } \\
\text { y análisis de } \\
\text { correlación } \\
\text { verbal. }\end{array}$ & $\begin{array}{l}\text { Estudio individual y de asociación entre cada dimen- } \\
\text { sión y subdimensiones de la IO abordadas desde las } \\
\text { encuestas: Disciplinas Io (Senge, 1990), componentes } \\
\text { de Albrecht (2003), inteligencia emocional; creación } \\
\text { y gestión del conocimiento organizacional, capacidad } \\
\text { de aprender y crear/innovar, capacidad de responder } \\
\text { y resolver problemas, capacidad de vigilar e interpre- } \\
\text { tar el entorno. } \\
\text { - Estudio de cada dimensión y subdimensiones de la IO } \\
\text { abordadas desde las entrevistas: Percepción del entorno } \\
\text { externo (vigilancia de la demanda, mercados, competi- } \\
\text { dores, proveedores, políticas gubernamentales, econo- } \\
\text { mía y sociedad, cultura y red organizacional), capaci- } \\
\text { dad de crear e innovar, toma de decisiones adaptativas } \\
\text { y generativas, acción de la organización (capacidad de } \\
\text { responder y resolver problemas), desempeño organiza- } \\
\text { cional y resultados innovadores (financiero, comercial } \\
\text { y con los clientes, procesos internos y aprendizaje y } \\
\text { crecimiento organizacional) y estudio de correlación } \\
\text { entre las dimensiones y subdimensiones de la Io. } \\
\text { Estudio comparativo y complementario entre los re- } \\
\text { sultados obtenidos por las encuestas y las entrevistas. }\end{array}$ \\
\hline
\end{tabular}

Continúa 


\begin{tabular}{|c|c|c|}
\hline $\begin{array}{l}\text { Fase } 3 \\
\text { Praxis de la inteligencia } \\
\text { organizacional. } \\
\text { (Desarrollo empírico: } \\
\text { estudio de la praxis que } \\
\text { atañe a la inteligencia } \\
\text { organizacional en } \\
\text { concordancia con el } \\
\text { trabajo de campo). }\end{array}$ & $\begin{array}{c}\text { Análisis } \\
\text { documental } \\
\text { y entrevistas } \\
\text { a todos los } \\
\text { líderes de la } \\
\text { organización. }\end{array}$ & $\begin{array}{l}\text { - Cada una de las dimensiones y subdimensiones de la } \\
\text { IO abordadas desde las entrevistas. } \\
\text { - Componente empírico emergente que hace parte de la } \\
\text { entrevista para identificar e incorporar en el modelo } \\
\text { de gestión de la inteligencia organizacional: ¿cómo } \\
\text { cree que Gráficas Modernas S.A. pueda incrementar } \\
\text { su inteligencia organizacional? } \\
\text { - Contexto empresarial. }\end{array}$ \\
\hline $\begin{array}{l}\text { Fase } 4 \\
\text { Desarrollo del } \\
\text { modelo de gestión } \\
\text { de la inteligencia } \\
\text { organizacional. } \\
\text { Desarrollo teórico- } \\
\text { empírico: proponer } \\
\text { un modelo de gestión } \\
\text { de la inteligencia } \\
\text { organizacional para } \\
\text { la empresa Gráficas } \\
\text { Modernas S.A., que } \\
\text { permita explicar } \\
\text { de forma teórica y } \\
\text { empírica la manera de } \\
\text { gestionar su inteligencia } \\
\text { organizacional. }\end{array}$ & $\begin{array}{c}\text { Análisis } \\
\text { documental, } \\
\text { revisión de } \\
\text { la literatura, } \\
\text { análisis de } \\
\text { encuestas a los } \\
\text { colaboradores } \\
\text { y análisis de } \\
\text { entrevistas } \\
\text { realizadas a los } \\
\text { líderes de la } \\
\text { organización. }\end{array}$ & $\begin{array}{l}\text { - Cada una de las dimensiones y subdimensiones de la } \\
\text { IO abordadas desde las entrevistas y encuestas. } \\
\text { - Componente empírico emergente de la Io para la em- } \\
\text { presa Gráficas Modernas S.A. }\end{array}$ \\
\hline
\end{tabular}

Fuente: Elaboración propia.

Las fases del proyecto de investigación establecidas en la metodología presentan en sus dimensiones una adecuada validación para los resultados. Estas también permiten identificar límites teóricos y de tipo empresarial, así como aportes en materia de investigación de la io en relación con la literatura administrativa y de cara al sector de artes gráficas. Estos elementos se describen a continuación:
- Validez: La metodología empleada en el proyecto de investigación brinda credibilidad en los resultados ya que, por un lado, la encuesta, como técnica de investigación utilizada, fue aplicada a la totalidad de la población (censo). De este modo se representa al total de los colaboradores de Gráficas Modernas S.A., lo que, en términos estadísticos, representa la ausencia de un error 
muestral. Por otra parte, la entrevista, a pesar de estar dirigida a un grupo específico de los colaboradores (aquellos con cargo directivo o que tienen subalternos), está orientada hacia la obtención de información del contexto de la gerencia. Así, los resultados que se derivan de esta técnica complementan o nutren los conseguidos con la encuesta. De acuerdo con la revisión de literatura, no es frecuente que estas dos técnicas de investigación sean usadas de manera conjunta en los estudios de diagnóstico de la Io.

- Límites: Esta investigación tiene límites teóricos y derivados del tipo de empresa en la que se realiza el estudio. Los primeros tienen que ver con el hecho de que en el proyecto se estudian y analizan los aportes desde la literatura administrativa relacionados únicamente con la inteligencia organizacional y no con sus componentes vistos de manera aislada. Esto teniendo en cuenta que la Io está estrechamente vinculada con otros temas administrativos por ser una acepción integradora y muy amplia en términos organizacionales. Los segundos se asocian con la imposibilidad de generalizar de forma directa los desarrollos, resultados y conclusiones conseguidos en el proyecto de investigación, hacia otras corporaciones de características similares o al sector empresarial como un todo. Esto, teniendo en cuenta que la amplitud del problema de investigación está centrada en el desarrollo de un modelo de gestión de la Io diseñado, específicamente, para la compañía Gráficas Modernas S.A. Sin embargo, una aplicación indirecta, es decir, a través de otros estudios con validez estadística y teórica, cuyos diseños sean desarrollados para tal fin, puede ser posible.

- Aportes: El proyecto de investigación aporta a la literatura administrativa al desarrollar un modelo de gestión de la Io para una empresa (Gráficas Modernas S.A.). Ello con base en la declaración de una definición propuesta para la IO. Adicionalmente, de acuerdo con el estado del arte revisado, no hay registro de desarrollos similares para una empresa del sector de artes gráficas de Cali (Colombia). Por último, el modelo de gestión de Io para la empresa en mención representa un aporte sobre el cual el cuerpo directivo de la organización puede emprender estrategias y planes de acción continuamente. Esto para mejorar las capacidades organizacionales y conducirlas hacia un plano superior, contribuyendo así a la obtención 
de mejores condiciones para avanzar en la búsqueda del éxito empresarial.

\section{RESULTADOS}

Como resultado fundamental de la investigación, en el presente capítulo se explica la manera en que fue desarrollado el modelo de gestión de la io propuesto para la compañía Gráficas Modernas S.A. Para ello se ha considerado necesario incorporar los siguientes cuatro apartados:

\subsection{Primer elemento para el desarrollo del modelo de gestión de la io}

Luego de un trabajo de investigación de la literatura administrativa se concluyó que la Io no cuenta en la actualidad con una definición universalmente aceptada. Es posible encontrar, en efecto, múltiples posiciones de diversos autores que se han dedicado al estudio de este campo. Sin embargo, se identifica la existencia de tres grandes consideraciones acerca de este concepto. La Io se vincula de esta manera con:

1. Una capacidad organizacional (Akgün, Lynn \& Byrne, 2003; Carro \& Carro, 2008; Castañeda, 2004; Choo, 1999; Cubillo, 1997; Erçetin, 2005; Glynn, 1996; Halal, 1997; Jung, 2009; Kalkan, 2005;
Kerfoot, 2003; Liao, Chang, \& Wu, 2010; Martínez, 2000; Más Basnuevo, 2005a, 2005b, 2006; McMaster, 1998; Méndez, 2012; Dibella \& Nevis, 1995; Ramos, 2007; Rodríguez, 2005, 2008; Román \& Díez, 2012; Romera, 2012; Schwaninger, 2001; Senge, 1990; Tarí \& García, 2009; Torres, 2002; Veryard, 2012).

2. Un proceso y un producto (De Rozas \& De Corcho, 2010; Estrada \& Salvador, 2007; López \& Correa, 2011; Nour Mohammad, Seyed Mohammad, \& Fatemeh, 2011).

3. Una función sistémica (Guerra, Molinillo, \& Bermúdez, 2009; Moreno, 2004; Orozco, 2001; Wilensky, 1967).

En la revisión de la literatura se hallaron estudios, provenientes de diferentes sectores organizacionales, en los que se define la Io teniendo en cuenta tanto los múltiples elementos teóricos desarrollados por diversos autores, como las particularidades de las empresas estudiadas. Entre los autores se destacan los trabajos de Cubillo (1997), Más Basnuevo (2006), McMaster (1998), Núñez (2004), Orozco (2006), Senge (1990) y Torres (2002).

Por otra parte, entre las organizaciones implicadas en los trabajos, se destacan la consultoría Biomundi, 
como centro de Io para la industria biofarmacéutica de Cuba (Orozco, 1999); la Universidad de los Andes (Viloria, 2003), Cementos Mexicanos CEMEX (Rodríguez, 2005), el Laboratorio Farmacéutico Oriente (Danger \& Sánchez de la Cruz, 2005), múltiples pequeñas y medianas empresas (Valecillos \& Quintero, 2007), Electrónica Arteche Hermanos S.A., Unión Fenosa, PriceWaterhouseCoopers, Siemens S.A., Grupo Telefónica, Grupo Santander (Claver \& Zaragoza, 2007), varias compañías metalmecánicas (Chirinos, Arévalo, $\&$ Chirinos, 2009), corporaciones locales de la Costa Oriental del Lago de Maracaibo (Quintero, Valecillos, \& Hernández, 2009), organizaciones del sector de TI de Banca Móvil (Fernández, 2011), el Hospital Universitario San Ignacio, como empresa del sector de salud en Colombia (Duque \& Castro, 2012) y las alcaldías de la Costa Oriental de lago de Maracaibo (Quintero, 2012).

Con todo, a pesar de la gran cantidad y variedad de literatura existente y de las diferentes organizaciones consideradas, no se hallaron estudios en los que se definiera la Io desde el contexto de empresas del sector de artes gráficas.

Así, a partir de los estudios anteriores se constata que a pesar de que la definición de la Io no cuenta con un concepto único por el cual se incline la comunidad académica, profesional y demás, su definición se fundamenta mayoritariamente en asimilarla como una capacidad sistémica organizacional que, de forma simultánea, se identifica como un proceso, un producto y una función. De esta manera, con base en las tres grandes posturas identificadas, y la revisión realizada, se propone la subsiguiente definición para la IO:

La Io se define como la capacidad sistémica del capital intelectual de una organización para aprender de su historia y del presente, construyendo día a día un futuro saludable sostenido, a través de un proceso creativo y efectivo en la percepción de su entorno interno y externo, de la creación y gestión del conocimiento y de la toma de decisiones, para garantizar una constante evolución adaptativa y generativa en favor de la consecución de la misión, los valores y la visión organizacional y el aporte al logro de una sociedad cada vez más inteligente.

De esta definición se desprendió la totalidad de la investigación realizada. Representó el primer elemento para el desarrollo del modelo de gestión de la io que fue elaborado para la empresa Gráficas Modernas S.A. Esta definición es novedosa en el sentido de que realiza una integración de las cuatro maneras de entender la Io (es decir, capacidad 
organizacional, proceso, producto y una función sistémica). Establece además un vínculo entre las organizaciones inteligentes y las sociedades al considerar que una sociedad llega a ser más inteligente si en ella se lleva a cabo una interacción positiva entre distintas organizaciones que permanentemente desarrollen su IO. Esto puede verse reflejado en unos resultados organizacionales satisfactorios que impacten positivamente el plano social.

\subsection{Componentes teóricos de la io}

$\mathrm{Al}$ ahondar en la investigación de la IO se encontró que algunos autores (Danger, 2005; Huber, Nadler, Gerstein, \& Shaw, 1998; Zerón, 2008) sugieren la existencia de ciertas condiciones necesarias para generarla. Entre estas se encuentran la cultura de aprendizaje, la motivación laboral, el clima laboral óptimo, un sistema de valores y de dirección coherente con el aprendizaje organizacional, el empleo consciente de medios para la gestión del conocimiento organizacional, la solución sistémica de problemas y la experimentación con nuevos enfoques para la obtención de saberes novedosos y provechosos.

Se identifican también otros elementos básicos relacionados con la IO. Entre estos se destacan:
- Una serie de características en las organizaciones con gran IO, vinculadas particularmente con la importancia de una adecuada gestión del conocimiento organizacional (North \& Rivas, 2008).

- Aspectos comunicacionales esenciales que toda entidad inteligente debe considerar en su gestión, como la claridad, la oportunidad, la seguridad, la validez, la adecuación y la extensibilidad (Arévalo, 2008 citado en González, 2012; Deagostini \& Comenzana, 2005).

- La sensación de mejorar la inteligencia, conocer el perfil de Io actual, tratar los asuntos prioritarios y controlar el progreso. Estos como principios de la IO (Mendelson \& Ziegler, 2004).

- La cognición, la memoria, el aprendizaje, la comunicación, el razonamiento y la cultura organizacional, en tanto componentes fundamentales de la Io (Chirinos, Arévalo, \& Chirinos, 2009).

También se encontró y estudió detalladamente una amplia variedad de propuestas de modelos teóricos de la IO realizadas en estudios precedentes. Entre estos se destacan los desarrollados por Albrecht (2002), Ashton \& Stacey (1995), Carpio (2010), Choo (1999), Fallas (2010), Falleta (2008), Halal(1997), Kalkan (2005), Lagerstam (1990), March y Olsen 
(1976), Martinet y Marti (1998), Más Basnuevo (2005a, 2005b), North y Rivas (2008), Orozco (1999), Orozco (2001), Rodríguez (1998), Senge (1990) y Castañon y Solleiro (1998).

En estos estudios es frecuente que se presenten componentes básicos como las capacidades organizacionales de aprender, crear e innovar, vigilar, la gestión del conocimiento organizacional, el liderazgo, la inteligencia emocional colectiva, la eficiencia y eficacia, el capital humano y un perceptible interés por alcanzar un futuro con éxito sostenido.

En la literatura se encuentran, finalmente, múltiples desarrollos de modelos de gestión de la Io para diferentes organizaciones de diversos sectores y tamaños. Entre estos se destacan los realizados acerca de:

- Dos grandes compañías de la ciudad de Zahedan, ubicada en Irán (Nour Mohammad, Seyed, \& Fatemeh, 2011)

- El Ministerio de Ciencia, Tecnología y Medio Ambiente (CITMA) de Cuba (Más Basnuevo, 2005b, 2006)

- El sector textil portugués (Pinheiro, Hernández, \& Raposo, 2007)

- La industria de la construcción (Jung, 2009)

- Instituciones de educación continua (Liao, Chang, \& Wu, 2010)
- La Facultad de Ciencias Económicas y Sociales de la Universidad de Zulia (Valecillos \& Quintero, 2009)

Sin embargo, como se ha indicado antes, no se identificaron estudios realizados en vinculación con la actividad de desarrollar modelos de gestión de la inteligencia organizacional, en particular, para empresas del sector de las artes gráficas.

\subsection{Componentes empíricos} desde el contexto empresarial de la compañía Gráficas Modernas S.A.

Como resultado del diagnóstico de la Io en la empresa Gráficas Modernas S.A. se logró identificar una serie de componentes empíricos propios de la organización. Estos se ajustan a las características y particularidades del sector de artes gráficas en Colombia. De esta manera, los principales factores empíricos que emergieron del estudio, y que resultaron vitales para el desarrollo del modelo de gestión IO, fueron los siguientes:

- Alto valor agregado en las soluciones: Un aspecto que ocupa de manera importante la atención de la dirigencia de la organización es la forma de garantizar que los productos de la organización, al 
interior del mercado de las artes gráficas, se caractericen por tener un alto valor agregado en el desarrollo del diseño estructural y los acabados. Ello como factor diferenciador. Además, en este componente de la IO cobra relevancia la gestión de la calidad en los servicios y las soluciones ofrecidas.

- Relaciones con otras organizaciones de interés: Parte de los factores de éxito de una empresa como Gráficas Modernas S.A., en este sector, se encuentra en la posibilidad de establecer relaciones interorganizacionales desde el entorno de acción. Esto para lograr beneficios colectivos derivados de una gestión adecuada en materia de información del sector que es considerada como valiosa (por ejemplo, las oportunidades de mejora y crecimiento económico empresarial en el mismo). Así, es esencial la actuación y la comunicación permanente con otras organizacionales decisivas como la Asociación Colombiana de la Industria de la Comunicación Gráfica (Andigraf), la Cooperativa de Impresores y Papeleros de Colombia (Coimpresores), la Cámara de Comercio, la Universidad Icesi, el Centro de Desarrollo Tecnológico para la Industria de la Comunicación Gráfica (Cigraf), el Servicio Nacional de Aprendizaje (Sena), entre otras.

- Motivación laboral desde los líderes: En el estudio se percibió que una adecuada motivación laboral incorporada dentro de la gestión de cada líder de la organización puede contribuir de manera importante a una IO superior para Gráficas Modernas S.A. De acuerdo con De Quijano y Navarro (1998), inspirados en Vroom (1964), la motivación se refiere al nivel de esfuerzo que una persona está dispuesta a hacer para llevar a cabo un trabajo. Sin embargo, de acuerdo con los planteamientos clásicos de Maslow (1991), la motivación está vinculada a una jerarquía de necesidades básicas a satisfacer que van desde las fisiológicas, pasando por las de seguridad, sociales y de estima, hasta llegar a las de autorrealización. De este modo, desde el plano organizacional es fundamental contar con una gestión permanente enfocada en el sostenimiento de la motivación laboral. Es necesario gestionar adecuadamente los esfuerzos individuales del talento humano con orientación hacia el logro de los objetivos organizacionales $\mathrm{y}$, en concordancia con esto, estar atentos a las diferentes necesidades que dan forma a la motivación. 
- Capacitación del talento humano: Se identificó que uno de los componentes necesarios para desarrollar de manera permanente el capital humano de la empresa es la realización de una serie de capacitaciones orientadas no solo al fortalecimiento de los conocimientos y las habilidades específicas de las funciones de cada cargo, sino también a una apropiada conductay convivencia con los demás colaboradores de la empresa. Esto puede contribuir a lograr un mejor clima organizacional. Cobran entonces una especial importancia instituciones educativas como el Servicio Nacional de Aprendizaje (Sena), así como los espacios universitarios de capacitación especializada y las asesorías y consultorías, entre otras.

- Sensibilización del personal orientada hacia el logro empresarial: Se reconoce la importancia de encaminar actividades enfocadas a concientizar y sensibilizar el talento humano en relación con la relevancia de cada una de las funciones que desempeñan. Estas deben contribuir a fructificar el desarrollo y crecimiento organizacional. Con esta sensibilización se podría prevenir la aparición de inconformidades, las cuales causan reprocesos y costos mayores a los proyectados.
- Espacios de participación: Una dimensión fundamental que es necesario desplegar desde la alta dirigencia de Gráficas Modernas S.A. son la generación y la diversificación de espacios de participación formales e informales. Estos son importantes para que los colaboradores puedan opinar sobre diferentes situaciones organizacionales, expresar sin temores sus ideas y pensamientos y crear un ambiente de diálogo enfocado hacia la resolución y respuesta de las situaciones organizacionales.

- Mejora e innovación tecnológica: La adquisición de maquinaria y equipo de trabajo que esté a la vanguardia desde el plano tecnológico es un prerrequisito básico dentro de la gestión interna de Gráficas Modernas S.A. Esto contribuye, entre otras cosas, a contar con una capacidad instalada, de producción sobresaliente, que responda con eficacia y eficiencia las soluciones que demandan los clientes. Esto es importante también para conseguir -mediante herramientas computacionales, programas actuales y otros instrumentos necesariosresultados destacados en materia de diseño estructural y gráfico, que incorporen el más alto valor agregado y la mayor calidad posibles. 
- Integración y sentido de pertenencia: La dirección de la organización requiere contemplar una serie de comportamientos y actividades que susciten en los colaboradores pensar que ellos son una parte activa y del mayor valor para la empresa. Con esto puede conseguir que se sientan integrados y tengan un sentido de pertenencia, en correspondencia con sus propósitos y visiones individuales.

Los anteriores elementos, que configuran las particularidades organizacionales de Gráficas Modernas S.A., son esenciales dentro del marco de estudio de la Io. Estos, por supuesto, son también de necesaria consideración para el desarrollo de un modelo de gestión de la Io en vinculación con esta empresa.

\subsection{Modelo de gestión de la IO para la compañía Gráficas Modernas S.A.}

Como resultado de la determinación de los principales factores teóricos que posibilitan la gestión y medición de la Io, que condujeron a la realización del diagnóstico de la IO en la empresa Gráficas Modernas S.A. y, posteriormente, a la identificación de los principales factores empíricos que emergieron de esta empresa de estudio, se procedió al desarrollo del modelo de gestión de la Io para esta compañía.

Se propone un modelo de gestión de la Io que consta de las siguientes dimensiones y subdimensiones:

- Las disciplinas planteadas por Senge (1990): Dominio personal, modelos mentales, visión compartida, aprendizaje en equipo y pensamiento sistémico.

- Las dimensiones consideradas por Albrecht (2002, 2003): La visión estratégica, el apetito por el cambio, corazón, alineación y congruencia y presión por el desempeño.

- La inteligencia emocional colectiva: Trabajo en equipos, capacidad de responder y resolver problemas, gestión del conocimiento organizacional y la capacidad de aprender e innovar como subdimensiones de esta.

- La creación y gestión del conocimiento organizacional: Aprendizaje y memoria organizacional, gestión de la comunicación y de la información como subdimensiones.

También se consideran la capacidad de aprender y crear/innovar, la acción de la organización (capacidad de resolver problemas o eficiencia, capacidad de responder o eficacia), percepción del entorno interno y externo (procesos y productos, colabo- 
radores, cultura y clima organizacional, mercados, competidores, proveedores, gobierno, situación económica y social, medio ambiente, cultura del país y red organizacional), toma de decisiones (adaptativas, generativas), desempeño organizacional y resultados innovadores (financiero, comercial y en el servicio al cliente, procesos internos, aprendizaje y crecimiento organizacional), sensibili- zación del personal; motivación laboral desde los líderes, espacios de participación, y mejora e innovación tecnológica. Otros elementos, que por su elevada importancia fueron incorporados también en el modelo de gestión fueron los valores, la misión y visión organizacional, así como las declaraciones de dirección y el alto valor agregado en las soluciones (ver figura 1).

Figura 1. Modelo de gestión de la Io desarrollado para la empresa Gráficas Modernas S.A.

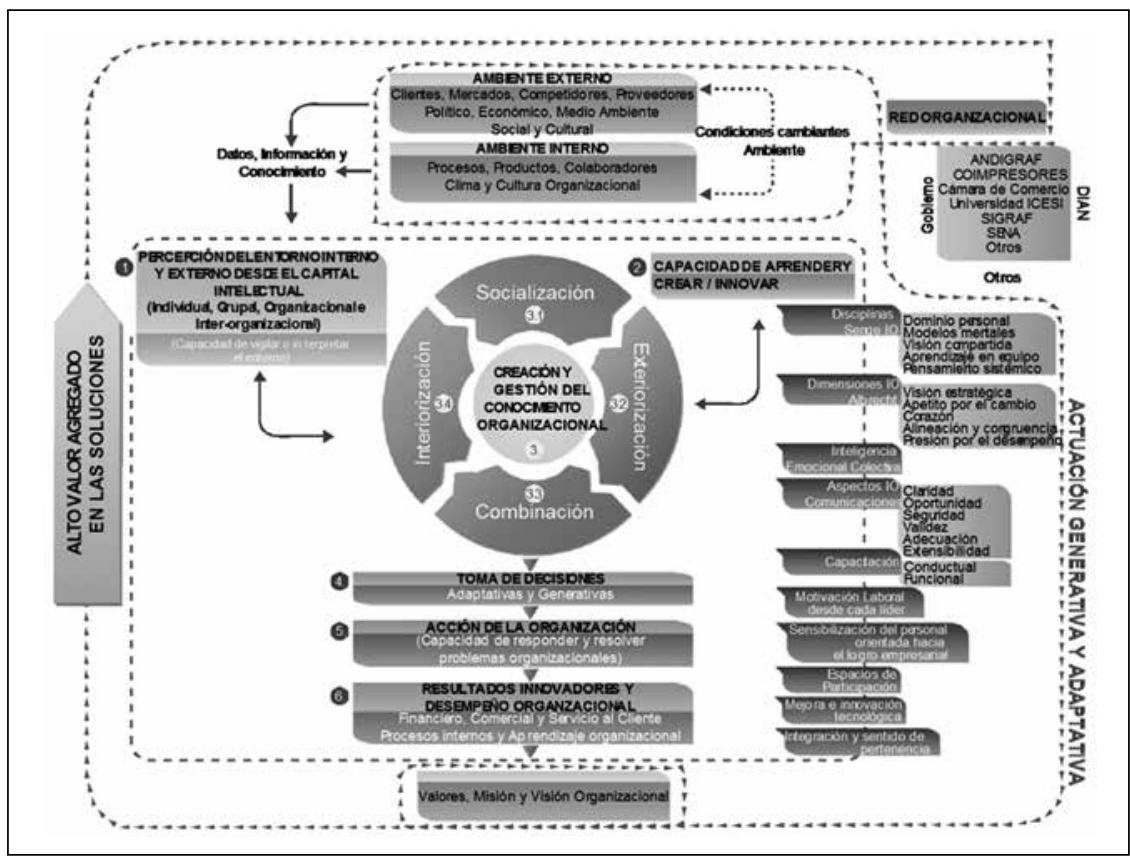

Fuente: Elaboración propia.

De acuerdo con la ilustración anterior, para lograr desarrollar continuamente la Io en Gráficas Moder- nas S.A., es necesario, en primer lugar, emprender un análisis profundo en el que el capital intelectual 
perciba — desde el nivel individual hasta el interorganizacional-el entorno en sus dos dimensiones:

1. Interna: Desde el nivel individual, grupal y organizacional de la empresa, estudiando variables como los procesos, productos, colaboradores, clima y cultura.

2. Externa: Desde el nivel organizacional e interorganizacional de la empresa, indagando sobre los clientes, mercados, competidores, proveedores, elambiente político, la economía, el medio ambiente, el componente social y cultural y las demás variables exógenas.

Este proceso, correspondiente a la primera dimensión del modelo (ver figura 1), y que mide la capacidad de vigilar e interpretar el entorno, deriva en la obtención de datos, información y conocimientos fundamentales. Estos son útiles como entrada a la creación y gestión del conocimiento organizacional (la tercera dimensión del modelo).

En este estudio el capital intelectual es entendido, en línea con lo sugerido por Jung (2009), como los recursos o activos tangibles e intangibles dentro de una organización que pueden contribuir a la Io. Estos se dividen en tres tipos de capital: humano $(\mathrm{CH})$, organizacional $(\mathrm{CO})$ y relacional $(\mathrm{CR})$. El primero se refiere al talento humano dentro de una organización que puede ser desplegado para adquirir y aplicar conocimiento para desempeñarse, responder o controlar el trabajo designado con los activos organizacionales disponibles. El segundo está asociado con los activos disponibles, excluyendo el $\mathrm{CH}$, para dar soporte a la realización de actividades organizacionales. El tercero, el capital relacional, combina el cH y co para realizar una actividad organizacional específica como, por ejemplo, cuando el diseñador industrial de Gráficas Modernas S.A. realiza una propuesta de un empaque para un cliente particular, en donde se requiere un capital humano (el personal) y un capital organizacional (la computadora, software y el plotter de corte).

En Gráficas Modernas S.A. la percepción del entorno se logra mediante una gama de actividades organizacionales, entre las cuales se pueden mencionar ferias nacionales e internacionales, seminarios, congresos de artes gráficas, Internet, revistas y libros especializados, encuestas de satisfacción del cliente, los puntos de venta. Esto, además de vínculos con Andigraf y otras entidades adjuntas a la red organizacional de Gráficas Modernas S.A., como las universidades, el gobierno, las actividades de planeación estratégica y los convenios empresariales para el cuida- 
do del medio ambiente, entre otros. Mientras, internamente es esencial monitorear cada uno de los procesos, productos, las actividades y el comportamiento de los colaboradores, el ambiente laboral y la cultura de la organización, de modo que se favorezca el aprendizaje, la creación y la gestión del conocimiento organizacional para la obtención de resultados empresariales sobresalientes.

Como producto del proceso de la percepción del entorno interno y externo, se obtienen valiosos datos, informaciones y saberes que representan las principales entradas para la creación y gestión del conocimiento organizacional. Sin embargo, otro componente o dimensión de la IO, de vital importancia para impulsar la creación y gestión del conocimiento organizacional, es el desarrollo permanente de la capacidad de aprender y crear e innovar. Esto a lo largo y ancho de la organización (la segunda dimensión del modelo de gestión). La capacidad de aprender se desarrolla encaminando actividades organizacionales que posibiliten la experimentación como 1) un modo de aprender, 2) el aprendizaje de los clientes internos y externos, 3) el aprendizaje de los procesos y productos que se realizan diariamente, 4) el aprendizaje junto a los demás colaboradores y 5) incitar a la socialización y rápida asimi- lación de información, destrezas y conocimientos que luego se evidencien en cambios positivos a nivel individual, grupal y organizacional.

Por su parte, la capacidad de crear e innovar se madura estimulando a los colaboradores - vía capacitación y entrenamiento o mediante una base de ideas creativas - hacia un pensamiento original. Se trata de lograr producir nuevas y prometedoras ideas de solución a los problemas, así como de involucrar la mejora continua, la creatividad y la innovación en las actividades relativas a los procesos, productos, servicios, proveedores y clientes internos y externos.

Una vez obtenidos los datos, la información y los conocimientos derivados de la percepción del entorno interno y externo (primera dimensión), y mediante el desarrollo de las capacidades de aprender y crear/innovar (segunda dimensión), se gesta el proceso de creación y gestión del conocimiento organizacional (tercera dimensión). Esto último se refiere a la manera en que la empresa se encarga de producir y administrar el saber organizacional, como base para la toma de decisiones. Es fundamental considerar la existencia de conocimientos tácitos y explícitos, así como identificar y gestionar cuatro patrones básicos alrededor de estos dos tipos de conocimientos para 
poder crear nuevos saberes (Nonaka \& Takeuchi, 2000): 1) socialización (paso de conocimiento tácito a tácito), 2) combinación (de explícito a explícito), 3) exteriorización (de tácito a explícito) y 4) interiorización (de explícito a tácito).

De acuerdo con Nonaka y Takeuchi (2000), el conocimiento tácito se refiere a aquel que está interiorizado en cada una de las personas, es difícil de formalizar y representa el punto de partida para la innovación. El conocimiento explícito, por su parte, es aquel que puede ser compartido y comunicado fácilmente mediante distintos medios comunicacionales. De esta manera, según los autores, en efecto, la generación de conocimiento organizacional sigue la siguiente espiral del conocimiento:

\section{Socialización (de tácito a tácito):}

Se refiere al conocimiento tácito que se comparte directamente de una persona a otra, conseguido mediante la observación, imitación y la práctica continua, es decir, justamente cuando se socializa el conocimiento. No obstante, la socialización es una manera muy limitada de creación de conocimiento, porque no se consigue una perspectiva sistemática del mismo y, dado que no se vuelve explícito, la compañía en general no lo puede aprovechar (ver numeral 2.1 en la figura 1).
2. Exteriorización (de tácito a explícito): Se presenta cuando se logra expresar por medios formales el conocimiento tácito, es decir, logrando que este sea explícito y, por lo tanto, divulgable a otras personas de la organización. Esto se da, por ejemplo, cuando el presidente ejecutivo de Gráficas Modernas S.A. prepara un informe gerencial en el que sintetiza la información de las distintas áreas e introduce un nuevo enfoque gerencial. Este lo hace según su conocimiento tácito, el cual ha adquirido mediante una amplia experiencia en el sector de las artes gráficas (ver numeral 2.2 en la figura 1).

3. Combinación (de explícito a explícito): Se da cuando una o varias personas combinan partes discretas de conocimiento explícito en otro completamente nuevo. Por ejemplo, cuando el presidente ejecutivo de Gráficas Modernas S.A. documenta formalmente el informe gerencial y lo traduce, por ejemplo, en procesos, manuales, instructivos, etc., dándole así forma a un producto organizacional que es público y útil para los demás colaboradores (ver numeral 2.3 en la figura 1).

4. Interiorización (de explícito a tácito): Una vez se comparte el nuevo conocimiento explícito, producto de la exteriorización y la 
subsiguiente combinación, los demás colaboradores comienzan a asimilarlo y a utilizarlo para ampliar y reformular el propio conocimiento tácito de cada uno. En otras palabras, el conocimiento explícito, al ser introducido en la base de saberes de las personas, se convierte en tácito (ver numeral 2.5 en la figura 1$)$.

El nuevo conocimiento tácito inicia por segunda vez la espiral, aunque en un nivel más alto dado el incremento previo en la base de conocimientos organizacionales (por esto se considera la analogía con un espiral ascendente). De esta manera, de nuevo se trasmite informalmente a otros colaboradores (socialización) se exterioriza y combina el nuevo saber emergente adquirido y, luego, se interioriza en los colaboradores. Así sucede de manera sucesiva, tal como se presenta en el modelo propuesto.

Una vez se haya creado el conocimiento organizacional, este se convierte en la base para dar inicio al proceso de toma de decisiones (cuarta dimensión). De acuerdo con Senge (1990), una organización inteligente aprende y continuamente expande su capacidad para crear su futuro. Es evidente que no basta con sobrevivir mediante un aprendizaje meramente adaptativo, sino que también es necesario vincularlo con un aprendizaje de tipo generativo. Por lo anterior se propone que el proceso de toma de decisiones de la dirección de Gráficas Modernas S.A. derive en decisiones de naturaleza tanto adaptativa como generativa. Las primeras se asocian con la capacidad de acomodarse o ajustarse a los cambios o altibajos del entorno dinámico, las segundas, con decisiones que repercuten en la generación, producción o creación de servicios, productos y procesos que alteren el entorno para bien de la organización.

Así, durante la toma de decisiones se deben sopesar las ventajas y las desventajas de las diferentes alternativas y escenarios para la resolución de problemas y de las eventuales situaciones organizacionales que sea necesario enfrentar. Esto, de acuerdo con Choo (1999), para que los resultados adaptativos y generativos resultantes sean los más convenientes para la empresa, no solamente en términos de desempeño organizacional, sino también en su proceso evolutivo en cuanto tal.

De esta manera, en línea con lo planteado por North y Rivas (2008), las decisiones generativas y adaptativas que se tomen desde la dirección de Gráficas Modernas S.A. podrán generar una serie de acciones organi- 
zacionales (quinta dimensión) que medirán, entre otras cosas, las capacidades empresariales de responder con eficacia y resolver con eficiencia los problemas organizacionales. En concordancia también con estos autores, la capacidad de respuesta ha de manifestarse ante variables como las nuevas tendencias del mercado, las ofertas de la competencia, la consulta de los clientes con rapidez y precisión, la introducción de nuevas funcionalidades o nuevos productos y servicios, la aparición de nuevos procesos, las inquietudes de los colaboradores y la formalización del conocimiento adquirido con los proveedores.

La capacidad de resolver problemas, por su parte, se despliega en lo relativo a los nuevos mercados, de manera conjunta con los competidores, anticipándose para resolver problemas y necesidades de los clientes. Se trata de poder resolverlos, dentro de un plazo adecuado y de manera efectiva y eficiente. Se despliega también en los procesos, los colaboradores y la cooperación de forma abierta y dinámica con los proveedores.

Finalmente, las acciones organizacionales ejecutadas desde Gráficas Modernas S.A. han de traducirse en unos resultados innovadores $\mathrm{y}$, luego, en un desempeño organiza- cional sobresaliente. Esto de acuerdo con lo planteado a nivel teórico por Kalkan (2005). Por consiguiente se hace necesario que la acción organizacional devenga en unos resultados innovadores y el desempeño organizacional satisfactorios (sexta dimensión). Ello en materia de resultados financieros y comerciales, de crecimiento respecto a años anteriores, frente a las metas presupuestales y al desempeño promedio del mercado.

Adicionalmente, la gestión de las necesidades del cliente en la etapa de preventa, venta y posventa ha de estar en unos niveles que genere una alta satisfacción. Los procesos internos deben procurar el control y aseguramiento de la calidad, así como la mejora e innovación en lo relativo al servicio al cliente, la eficiencia y la eficacia. Es necesario también que se presente un aprendizaje y crecimiento organizacional favorable en materia de tecnología, clima laboral y desarrollo del talento humano. Esto en coherencia con la declaración de los valores, la misión y la visión de empresa.

Todo ello sigue una espiral de Io que nunca deja de moverse y ampliarse. Esto representando en sí misma una actuación generativa y adaptativa permanente, la cual tiene en cuenta que actividades como el apren- 
dizaje, la creatividad y efectividad en la percepción del entorno y en la creación y gestión del conocimiento siempre se deben ajustar y afinar a través del tiempo. Gracias a esto se hace posible lograr, mediante acciones organizacionales sometidas previamente a un proceso inteligente de toma de decisiones, una contante reinvención de la empresa. Ello de cara a unos resultados innovadores y sobresalientes, con alto valor agregado en los productos y un desempeño organizacional exitoso sostenido. Esta es una cualidad esencial de una empresa con elevada IO.

\subsection{Características esenciales del modelo de gestión de la 10 propuesto}

Las principales características del modelo de gestión Io desarrollado en el marco de esta investigación son:

- Es un modelo de gestión dinámico, fundamentado en una espiral de la to que representa la importancia de involucrar en el interior de la organización una actuación adaptativa frente a los cambios provenientes del entorno. Este también insiste en la necesidad de participar como un organismo promotor de acciones que generen cambios positivos en el medio en que esta se desempeña, lo que permite garantizar una evolución organizacional permanente de cara a la sostenibilidad en el futuro.

- Considera una serie de capacidades y dimensiones, entradas, procesos y salidas, que permiten modelar acciones organizacionales. Estas coadyuvan al avance hacia el logro de una organización con Io superior. Ello se contempla en afinidad con el logro de la misión, la visión, los valores y el éxito empresarial a través del tiempo. El modelo aporta a la función capital de Gráficas Modernas S.A. de desarrollarse y prosperar como un sistema organizacional dinámico.

- Reconoce las particularidades del contexto empresarial actual para mejorar la Io de Gráficas Modernas S.A. Invita a considerar aspectos como las capacitaciones en el componente humano y técnico, la motivación laboral desde los líderes, la sensibilización del personal orientada al logro empresarial, la conveniencia de suscitar espacios de participación, mejora e innovación tecnológica y de desarrollar la creatividad, la integración y el sentido de pertenencia.

- Identifica grupos de interés del sector de artes gráficas consideradas hoy como instituciones clave, entidades relevantes e influyentes para el crecimiento empresarial 
de Gráficas Modernas S.A. Entre ellas se destacan Andigraf, la Cámara de Comercio, el CIGRAF, la Universidad Icesi, el Sena, Coimpresores, el Gobierno de Colombia y la Dirección de Impuestos y Aduanas Nacionales de Colombia (DIAN). Además, se esquematiza la incorporación de nuevas entidades y grupos de interés que permitan solidificar y madurar una completa red organizacional en constante comunicación e interacción sistémica. Esto para lograr la materialización de beneficios colectivos superiores para la empresa Gráficas Modernas S.A.

- Expresa los diferentes ámbitos por los cuales se realiza la vigilancia del entorno externo a la organización: cultural, económico y social, los mercados, los competidores, los proveedores, las políticas de gobierno, el medio ambiente y la cultura del país.

- Representa un paradigma en el que las capacidades organizacionales de aprender, crear/innovar, vigilar e interpretar el entorno interno y externo, desde el capital intelectual, configuran los principales catalizadores del núcleo (es decir, la creación y gestión del conocimiento organizacional) del modelo de gestión de la IO.
- El alto valor agregado aparece como un elemento que se desplaza en todas las dimensiones, procesos, productos y la red organizacional del modelo de gestión de la Io. Esto debido a que es precisamente este factor el que hace de Gráficas Modernas S.A. una empresa reconocida y diferente a las demás dentro de su sector de actividad.

\section{CONCLUSIONES}

Como resultado de la investigación realizada en el interior de la literatura administrativa, en primer lugar, no fue posible verificar la existencia una definición universalmente aceptada de la Io (un tema de estudio que en sí mismo es muy amplio), ni desde la academia ni desde el sector empresarial. Esta, sin embargo, se ha identificado como un proceso, un producto, una función del sistema organizacional y una capacidad organizacional que es posible desarrollar constantemente.

Con base en estos elementos y la consideración de numerosos estudios relativos al tema es posible comprender la IO como la capacidad organizacional de una entidad para percibir el entorno interno y externo que la rodea y tomar las decisiones más adecuadas posibles. Esto a través de un procesamiento 
minucioso de la información y el conocimiento. Con ello se generan nuevos flujos de estos elementos y de capacidades de aprendizaje en un nivel superior, los cuales se enfocan hacia el sostenimiento y desarrollo exitoso de la organización en el tiempo.

Teniendo en cuenta que no fue posible identificar publicaciones en materia administrativa que propusieran modelos de gestión de la IO particulares para empresas del sector de las artes gráficas en Colombia, el modelo de gestión de la IO desarrollado para Gráficas Modernas S.A. representa, en sí mismo, un aporte a la academia y al ámbito organizacional. En este sentido, el presente trabajo hace un aporte teórico-empírico encaminado hacia la mejora de la io y es útil a la búsqueda del éxito organizacional de una compañía perteneciente a un sector empresarial relativamente poco explorado en este campo.

Como resultado de la identificación de los principales factores teóricos que posibilitan la gestión y valoración de la IO, la realización del diagnóstico de esta variable en la empresa Gráficas Modernas S.A. y el establecimiento de los principales factores empíricos que emergieron de la organización se desarrolló un modelo de gestión de la IO. Este representa un paradigma organizacional útil para mejorar día a día en dicho aspecto. Este modelo consta de los siguientes componentes: las disciplinas de Senge (1990), las dimensiones planteadas por Albrecht (2002), la inteligencia emocional colectiva, la creación y gestión del conocimiento organizacional, la capacidad de aprender y crear/innovar, la acción de la organización, la percepción del entorno interno/externo, la toma de decisiones adaptativas y generativas, el desempeño organizacional y los resultados innovadores, la sensibilización del personal, la integración y el sentido de pertenencia, la motivación laboral desde los líderes, la consideración de la red organizacional, los espacios de participación, los aspectos comunicacionales, la capacitación conductual y funcional, la mejora e innovación tecnológica, los valores, misión y visión organizacional y el alto valor agregado en las soluciones.

Finalmente, hay varias maneras en que se puede desarrollar la io en la empresa Gráficas Modernas S.A. Estas son 1) realizar mayor capacitación en el componente humano y técnico, 2) fortalecer con más intensidad la motivación laboral desde los líderes, 3) trabajar más en la sensibilización del personal, 4) general un programa de sugerencias/recomendaciones de mejora desde los 
colaboradores, 5) hacer de la empresa un espacio más participativo, generando más ambientes para hacer que todos puedan realizar sugerencias, 6) mejora tecnológica, 7) desarrollar la creatividad en las actividades de cada colaborador, 8) tener en cuenta la capacidad instalada para la toma de decisiones relacionadas con trabajos de clientes y 9) generar una mayor integración y sentido de pertenencia hacia la empresa.

\section{REFERENCIAS}

Ashton, W., \& Stacey, G. (1995). "Technical intelligence in business: understanding technology threats and opportunities". Journal of Technology Management, 10(1), 79-104.

Akgün, A. E., Lynn, G. S. \& Byrne, J. C. (2003). Organizational learning: A sociocognitive framework. Human Relations, 56 (7), 839-868.

Albrecht, K. (2002). The power of mindsatwork: Organizationalintelligence in action. Nueva York: AMACOM.

Albrecht, K. (2003). Organizational intelligence survey. Sídney: Australian Institute of Management.

Carpio, I. (2010). Modelo de organización inteligente. Lima: Universidad Nacional de Ingeniería.
Carro Cartaya, J. C., \& Carro Suárez, J. R. (2008). La inteligencia empresarial y el sistema de gestión de calidad ISO 9001: 2000. Ciencias de la Información, 39(1), 31-44.

Castañeda, D. I. (2004). Estado del arte en aprendizaje organizacional, a partir de las investigaciones realizadas en facultades de psicología, ingeniería industrial y administración de empresas en Bogotá, entre los años 1992 y 2002. Acta Colombiana de Psicología, 11, 23-33.

Castañón, R., \& Solleiro, J. L. (1998). Evaluación de un sistema de monitoreo ecnológico (SMT). XIX Simpósio de Gestao da Inovaao Tecnológica, Sao Paulo.

Chirinos, A. A., Arévalo Cohen, F. H., \& Nava, C. (2009). Inteligencia organizacional y tecnologías de información en las empresas metalmecánicas. MT2 Nava et al SinncO. Recuperado de http://www.concyteg.gob.mx/formulario/MT/MT2009/MT2/SESION2/MT22_ANAVA_034.pdf Choo, C. W. (1999). The knowing organization: How organizations use information to construct meaning, create knowledge, and made decisions. Oxford: Oxford University Press.

Claver, E., \& Zaragoza, P. (2007). La dirección de recursos humanos en 
organizaciones inteligentes. Una evidencia empírica desde la dirección del conocimiento. Investigaciones Europeas de Dirección y Economía de la Empresa, $13,55-73$.

Cubillo, J. (1997). La inteligencia empresarial en las pequeñas y medianas empresas competitivas de América Latina: algunas reflexiones. Ciência da Informação, 26(3), 260-267.

Danger, Y. (2005). ¿Qué le impide a su empresa convertirse en una organización inteligente? Sociedad y Economía, 108, 554-569.

Danger, Y., \& Sánchez de la Cruz, S. (2005). ¿Es posible que la empresa Laboratorio Farmacéutico Oriente pueda convertirse en una organización inteligente? Dirección de Información Cientifico-Técnica Universidad de Oriente, Santiago de Cuba, CUBA, pp. 570-585.

Deagostini, A., \& Comenzana, F. (2005). Rol de las nuevas tecnologías de la información y la comunicación en la inteligencia organizacional aplicada a una pequeña y mediana empresa. Montevideo: Universidad Católica de Uruguay.

De Quijano, S. D., \& Navarro, J. (1998). Un modelo integrado de la motivación en el trabajo: Conceptualización y medida. Revista de Psicología del Trabajo y de las Organizaciones, 14(2), 193-216.
De Rozas, M., \& de Corcho León, L. (2010). Herramientas de inteligencia empresarial para el desarrollo de la innovación. Caso Saude. Ingeniería Industrial, 31(1), 1-7.

Dibella, A., \& Nevis, E. C. (1998). How organizations learn: an integrated strategy for building learningcapability. San Francisco: Jossey-Bass.

Duque, Y. V., \& Castro, A. G. (2012). Organizaciones inteligentes: evidencia empírica de una organización que aprende en Colombia (Hospital Universitario San Ignacio). Universitas Médica, 53(2), 126-143.

Erçetin, Ş. Ş. (2005). The abilities related to the organizational intelligence and their action dimensions at schools. Research for Educational Reform, 10(2), 3-18.

Estrada, R. A., \& Salvador, C. (2007). La inteligencia empresarial como herramienta de apoyo de la dirección estratégica. $A d$ ministración y Organizaciones, 10(19), 75-100.

Fallas, S. L. (2010). Un sistema de inteligencia interno para la organización inteligente. Ciencias Económicas 28(2), 481-524.

Falletta, S. V. (2008). Organizational intelligence surveys. Training \& Development, 62(6), 52-58.

Fernández, C. A. (2011). Propuesta de cambio para el desarrollo de una organización inteligente 
verde de ті Bancaria, basada en redes inteligentes ecológicas, banca móvil y ética organizacional para la Banca Universal. Negotium, 7(20), 82-112.

Glynn, M. A. (1996). Innovative genius: A framework for relating individual and organizational intelligences to innovation. Academy of management review, 21(4), 1081-1111.

González, F. (2012). Comunicación $e$ inteligencia organizacional como pilares de una gestión efectiva. Recuperado de http://www. gestiopolis.com/administracionestrategia-2/comunicacion-e-inteligencia-organizacional-comopilares-de-una-gestion-efectiva. htm.

Guerra, L., Molinillo, S., \& Bermúdez, G. (2009). Inteligencia organizacional como elemento dinamizador de configuraciones organizativas competitivas del sector empresarial. Espacios. Espacios, 30(3), 10-12.

Halal, W. E. (1997). Organizational intelligence: What is it, and how can managers use it? Recuperado de http://www.strategy-business. com/article $/ 12644$ ?gko $=4 a 546$

Jung, Y. (2009). An approach to organizational intelligence management (a framework for analyzing organizational intelligence within the construction process) (Tesis de doctorado inédita, Facultad del Instituto Politécnico de Virginia y Universidad del Estado, Blacksburg, EEUU).

Kalkan, V. D. (2005). Organizational intelligence: Antecedents and consequences. Journal of Business \& Economics Research (JBER), 3(10), 43-54.

Kerfoot, K. (2003). On leadership organizational intelligence/organizational stupidity: The leader's challenge. Nursing economics, 21(2), 91-93.

Lagerstam C. (1990). The theory of business intelligence: the intelligence process. En: C. Blaise, N. Tudor-Silovic (eds.). The knowledge industries: levers of economic and social development in the 1990s. Londres:Aslib.

Liao, S. H., Chang, W. J., \& Wu, C. C. (2010). An integrated model for learning organization with strategic view: Benchmarking in the knowledge-intensive industry. Expert Systems with applications, 37(5), 3792-3798.

López, G., \& Correa, M. (2011). Fuentes de información e inteligencia organizacional en investigación. El caso de la Universidad Tecnológica de Pereira. Cuadernos de Administración. 24(42), 231-252.

Martínez, C. E. (2000). Gerencia de las organizaciones inteligentes. Innovar, 15, 64-69.

Martinet, B., \& Marti, Y. M. (1995). L'intelligence économique: les 
yeux et les oreilles del'entreprise. París: Editions d'Organisation.

March, J., \& Olsen, J. P. (1976). Ambiguity and choice in organizations. Universitetsforlaget: Bergen.

Más Basnuevo, A. (2005a). Antecedentes y situación actual de los conceptos y métodos para el desarrollo de la inteligencia organizacional. Acimed, 13(4), 1-1.

Más Basnuevo, A. (2005b). Modelo para el desarrollo de un sistema de inteligencia organizacional en la delegación del Ministerio de Ciencia, Tecnología y Medio Ambiente en Holguín. ACIMED, 13(6), 1-27.

Más Basnuevo, A. (2006). Implementación de un modelo de desarrollo de la inteligencia organizacional en las esferas de actuación de la Delegación del CITMA en Holguín: primera etapa. Ciencias de la Información, 37(1), 45-58.

Maslow, A. H. (1991). Motivación y personalidad. Madrid: Díaz de Santos.

McMaster, M. (1998). Organizational intelligence (adaptada de una charla dada a la Industrial SocietyLondres, abril de 1998). Knowledge Based Development. The Industrial Society. Recuperado de http://www.parshift.com/ Speakers/Speak011.htm

Mendelson, H., \& Ziegler, J. (2004). Los 4 principios de la empresa inteligente: reinventar la empresa en la era de la información. Barcelona: Deusto.

Méndez, R. (2012). Organización inteligente. Recuperado de http:// www.rogermendez.es.t1/*-LaOrganizaci\%F3n-Inteligente. $\mathrm{htm}$ empresa inteligente. LibrosEnRed

Moreno, C. (2004). La inteligencia organizativa como dinamizador del capital intelectual. PUZZLE: Revista Hispana de la Inteligencia Competitiva, 3(14), 4-10.

Nonaka, I., \& Takeuchi, H. (2000). La empresa creadora de conocimiento. Gestión del conocimiento, p.1-9.

North, K., \& Rivas, R. (2008). Gestión del conocimiento. Una guía práctica hacia la empresa inteligente. Libros en Red: Colección: Negocios, Empresa y Economía.

Nour Mohammad, Y., Seyed Mohammad, S., \& Fatemeh, A. (2011). The relationship between organizational intelligence and strategic thinking. Journal of Theorical and Applied Information Technology, 31(1), 60-73.

Núñez, P. (2004). La gestión de la información, el conocimiento, la inteligencia y el aprendizaje organizacional desde una perspectiva socio-psicológica. Acimed, 12(3), 1-1.

Orozco, E. (1999). Organizational intelligence in the biopharma- 
ceutical industry. Ciência da Informação, 28(1), 59-66.

Orozco, E. (2001). El lugar de la inteligencia empresarial en el entorno conceptual de la gestión del conocimiento. Evolución en Cuba. El profesional de la información, 10(7), 14-22.

Pinheiro, P., Hernández, R., \& Raposo, M. (2007). La inteligencia organizativa de las empresas del sector textil portugués (Ponencia presentada al XXI Congreso Anual AEDEM, Empresa global y mercados locales, Madrid, España).

Quintero de Faría, N. M. (2012). Desempeño gerencial de las alcaldías de la costa oriental del lago de Maracaibo enfocado en las organizaciones inteligentes. Negotium, 8(22), 4-33.

Quintero, N., Valecillos, C., \& Hernández, G. (2009). Estrategias para fortalecer el dominio personal basado en el enfoque de las organizaciones inteligentes, en las corporaciones localdes, de la costa oriental del lago de Maracaibo. Negotium, 5(14), 32-48.

Ramos, I. (2007). The organizational mind: A comprehensive framework for the intelligent organization. Minho: University of Minho, Information System Department.

Rodríguez, M. (1998). La inteligencia tecnológica: Elaboración de mapas tecnológicos para la identificación de líneas recientes de investigación en materiales avanzados y sinterización (tesis doctoral, Universidad Politécnica de Cataluña, Barcelona).

Rodríguez, J. R. (2005). Ventajas competitivas de ser una organización inteligente; el caso de $\mathrm{Ce}$ mex. El Cotidiano, 20(130), 4650.

Rodriguez, Y. (2008). Gestión de información e inteligencia: integración en los contextos organizacionales. ACIMED, 17(5), 1-10.

Roman, M., \& Díez, E. (2012). Las organizaciones educativas aprenden ¿Cómo? (II).Aprendizajeinstitucional constructivo, significativo y mediado. Recuperado de http://martiniano.editorialconocimiento.cl/wp-content/ uploads/2009/05/las-organizaciones-educativas-aprenden-ii.doc

Romera, X. (2012). La inteligencia organizacional. Recuperado de http://blog.xavierromera. com $/ ? \mathrm{p}=5$

Senge, P. M. (1990). The fifth discipline: The art and practice of the learning organization. Nueva York: Doubleday.

Schwaninger, M. (2001). Intelligent organizations: An integrative framework. Systems Research and Behavioral Science, 18, 137-158. Tarí, J. J., \& García, M. (2009). Dimensiones de la gestión del 
conocimiento y de la gestión de la calidad: una revisión de la literatura. Investigaciones Europeas de Dirección y Economía de la Empresa, 15(3), 135-148.

Torres, A. (2002). El profesional de la información en la inteligencia organizacional. ACIMED, 10(5), 1-9.

Valecillos, C., \&Quintero, N.(2009). Modelo de gestión basado en el enfoque de las organizaciones inteligentes y las demandas del currículo integral caso: Facultad de Ciencias Económicas y Sociales de Luz. Negotium, 4(12), 63-85. Valecillos, C. A., \& Quintero, N. (2007). Enfoque de las organizaciones inteligentes en la implementación de nuevas técnicas de dirección en las pequeñas $\mathrm{y}$ medianas empresas (pymes).
Revista de Ciencias Sociales, XIII(2), 278-289.

Veryard, R. (2012). Organization intelligence. Recuperado de www. cbdiforum.com.

Viloria, N. (2003). La Universidad de Los Andes como organización inteligente. Actualidad Contable FACES. 6(6), 59-69

Vroom, V. H. (1964). Work and motivation. San Francisco: JosseyBass.

Wilensky, H. (1967). Organizational intelligence: Knowledge and policy in government and industry. American Sociological Review, 33(1), 131-132.

Zerón, F. M. (2008). Empresas inteligentes: moda o necesidad. Contribuciones a la Economía. Recuperado de http://www.eumed.net/ce/2008a/ 